This item was submitted to Loughborough's Research Repository by the author.

Items in Figshare are protected by copyright, with all rights reserved, unless otherwise indicated.

\title{
Homoclinic orbits in the near-integrable double discrete sine-Gordon
}

\section{equation}

PLEASE CITE THE PUBLISHED VERSION

LICENCE

CC BY-NC-ND 4.0

\section{REPOSITORY RECORD}

Rothos, Vassilios M.. 2019. "Homoclinic Orbits in the Near-integrable Double Discrete Sine-gordon Equation". figshare. https://hdl.handle.net/2134/682. 


\title{
Homoclinic Orbits in Near-Integrable Double Discrete sine-Gordon Equation
}

\author{
Vassilios M. Rothos* \\ Department of Mathematical Sciences \\ Loughborough University \\ Loughborough LE11 3TU UK \\ E-mail: V.M.Rothos@lboro.ac.uk
}

March 14, 2001

\begin{abstract}
We establish the existence of homoclinic orbits for the near-integrable double discrete sine-Gordon (dDSG) equation under periodic boundary conditions. The hyperbolic structure and homoclinic orbits are constructed through the Bäcklund transformation and Lax pair. A geometric perturbation method based on Mel'nikov analysis is used to establish necessary criteria for the persistent of temporally homoclinic orbits for the class of dDSGequations with dissipative perturbations.
\end{abstract}

$1991 \mathrm{MSC} 46,58 \mathrm{~F} 07$

PACS 02.30Ks, 02.40Vh, 05.45.-a

${ }^{*}$ Supported by a TMR postdoctoral fellowship No. ERBFMBICT983236 of Commission of the European Communities, and an EPSRC Grant No. GR/R02702/01. 


\section{Contents}

1 Introduction $\quad 3$

2 Formulation of the problem $\quad 4$

3 Integrability and Homoclinic Orbits $\quad 6$

3.1 Integrable Background $\ldots \ldots \ldots \ldots \ldots \ldots \ldots$

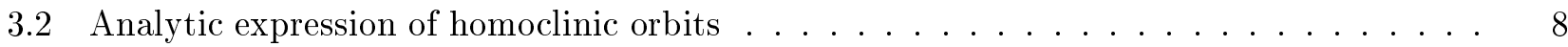

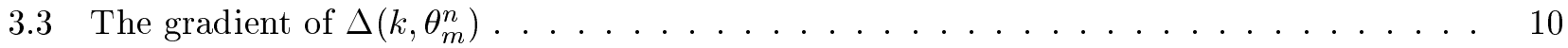

4 Homoclinic Orbits for perturbed dDSG equation $\quad 12$

4.1 Linearized analysis . . . . . . . . . . . . . . . . . . . . . . . 12

4.2 Mel'nikov method . . . . . . . . . . . . . . . . . . . . . 14

5 Conclusions $r$ 


\section{Introduction}

In recent years there has been remarkable progress on discrete integrable systems and associated difference equations. This paper is concerned with the existence of homoclinic orbits of the near-integrable double discrete sine-Gordon equation. A starting point is an integrable lattice version of the SG equation, which is a nonlinear partial difference equation $(\mathrm{P} \Delta \mathrm{E})$, i.e a system in which both the spatial-as well as the time-variable is discrete. Discrete space-time model in soliton theory have acquired a prominent role of mathematical physics. The first examples of such models were proposed by Hirota [10] as discrete analogues of the major continuum soliton models. Subsequent development was carried on mostly by the Dutch group (see Nijhoff and Capel [18] and references therein). The lattice sine-Gordon equation (double discrete sine-Gordon (dDSG)) that we want to investigate in this paper was first presented by Hirota [10] and therefore sometimes referred to as the Hirota equation. The particular discretization scheme of Hirota is used by Orfanidis [19] to study th discrete nonlinear $\sigma$-model from group-theoretical viewpoint. We show that the dDSG equation with periodic boundary conditions in the discrete space admits solutions which are homoclinic in the "discrete" time to the hyperbolic fixed point. We consider here the dDSG as a discrete dynamical system-a mapping with two indexes $n$ and $m$. For a specialist in dynamical systems, the Hirota equation gives a family of examples of symplectic maps of finite dimensional phase space so-called standard map, [3]. Also, the dDSG plays an important role in the discrete geometry. The continuum limit describes surfaces with constant negative Gausian curvature. The dDSG (classical) model possesses a nice geometric interpretation which parallels the continuum theory to surprising extent [4], and which provides a most useful guiding principle for the analysis of the model in geometric view point.

We present necessary criteria for the persistence of homoclinic structure of the dDSG equation with small dissipative perturbations, tending to the continuous perturbed SG equation for which we have established the persistence of homoclinic orbits and its temporal chaotic behaviour, (see [21] for geometric approach, [16] for analytic approach and [15] for numerical investigation). With the method being developed here we exploit two facts: first we investigate the existence of homoclinic orbits associated to a hyperbolic fixed point of the unperturbed dDSG through a Bäcklund transformation and second we establish the persistence of these orbits under small dissipative perturbations. Our results are therefore interested for the discrete K-surface with small perturbation (numerical simulations of discrete geometry), related to the new directions of the combination lattice equations and non-integrability criteria. We note that the dynamics of dDSG equation is very close to the continuum model. If the underlying dimension of some system under consideration is infinite (for example SG model), we are faced with two difficulties: first the techniques are largely unavailable for performing the qualitative analysis and second the phenomena occurring can be considerably more complicated. To resolve this apparent intractability of higher dimensional problems, techniques involving reductions to lower dimensional space are very attractive. Recently, Haller has been developing an alternative prespective, based on the existence of multi-pulse homoclinic solutions near resonance, which has applied to finite dimensional spatial discretizations of the perturbed NLS equations and to the pde (see [9] and references there in).

The paper is organized in the following manner. In Section 2, we formulate the problem. In section 3 , we review some basic concepts of the dDSG as a nonlinear P $\Delta \mathrm{E}$ and its relationship with discrete K-surfaces, we present the Lax pair of dDSG and find an analytic expression of the homoclinic orbits through the Bäcklund transformation. We obtain an explicitly formula for the gradient of the individual constant of motion evaluated on the homoclinic orbits. In Section 4 we derive necessary conditions for the persistence of homoclinic solutions of dDSG equation under dissipative perturbations based on the Mel'nikov analysis and geometric arguments of our problem. 


\section{Formulation of the problem}

The sine-Gordon (SG) equation

$$
\mathrm{u}_{x x}-\mathrm{u}_{t t}=\sin \mathrm{u}
$$

is one of the simplest integrable PDE with many physical and mathematical applications and admits solutions for very special initial data and $2 \pi$-periodic boundary conditions in space which are homoclinic in time. These homoclinic structures persist under dissipative perturbations and the near-integrable equation possesses temporal chaotic behaviour [21].

When we integrate numerically the SG equations, one considers the spatial variable to be a lattice of points, so the objects of study are sets on nonlinear ordinary differential equations with time remaining a continuous independent variable. Such problems have a venerable place in the nonlinear waves studies going back to Frenkel-Kontorova model (discrete in space version of the sine-Gordon equation), Fermi-Pasta-Ulam model. In the recent studies of the nonlinear dynamics of lattice systems consisting of coupled oscillators attention was paid to time-periodic and spatially localised excitations-discrete breathers-a prototype of such nonlinear lattice is represented by the discrete NLS equations. Dealing with the discrete lattice structure and thus with a network of coupled oscillators one faces the fact that most nonlinear lattice systems are non-integrable ([14] and references therein).

One of the aspects that make integrable equations very special is that are can find discrete analogues of these equations, sine-Gordon, $\mathrm{KdV}$ for instance, tending to the original continuous equations in special limits and that are integrable themselves. These discrete analogues can be either ordinary or partial difference equations (P $\Delta \mathrm{Es}$ ) like MacMillan map [8] or mixed differential-difference equations like Ablowitz-Ladik [1] and Toda lattice [23].

We consider the integrable discrete analogue of the equation (2.1):

$$
\left(1-\frac{h^{2}}{4}\right) \tan \frac{1}{4}\left(\theta_{m+1}^{n}+\theta_{m-1}^{n}\right)=\left(1+\frac{h^{2}}{4}\right) \tan \frac{1}{4}\left(\theta_{m}^{n+1}+\theta_{m}^{n-1}\right)
$$

as a discrete dynamical system and we establish the persistence of its homoclinic structure under small dissipative perturbations.

Equation (2.2) (first appeared in Hirota [10]) is a complete integrable discretization of the sineGordon equation (2.1), where $\theta_{m}^{n}:=\mathrm{u}(m h, n h)$ denotes the approximation of $\mathrm{u}(x, t)$. We rewrite $(2.2)$ in the form:

$$
\theta_{m}^{n+1}+\theta_{m}^{n-1}=\theta_{m+1}^{n}+\theta_{m-1}^{n}+2 \mathrm{i} \ln \left\{\frac{1+\frac{h^{2}}{4} \exp \left[\frac{\mathrm{i}}{2}\left(\theta_{m+1}^{n}+\theta_{m-1}^{n}\right)\right]}{1+\frac{h^{2}}{4} \exp \left[\frac{-\mathrm{i}}{2}\left(\theta_{m+1}^{n}+\theta_{m-1}^{n}\right)\right]}\right\}
$$

or using the identity $\operatorname{Arg}(z)=\vartheta=\frac{1}{2 \mathrm{i}} \ln \left(z \bar{z}^{-1}\right), z=r \mathrm{e}^{\mathrm{i} \vartheta}$, we rewrite the equation (2.3) as follows:

$$
\theta_{m}^{n+1}+\theta_{m}^{n-1}=\theta_{m+1}^{n}+\theta_{m-1}^{n}+2 \mathrm{i} \operatorname{Arg}\left\{1+\frac{h^{2}}{4} \exp \left[\frac{\mathrm{i}}{2}\left(\theta_{m+1}^{n}+\theta_{m-1}^{n}\right)\right]\right\}
$$

We consider the above equations (2.3) or (2.4) as a discrete evolution equation with respect to index $n$ (in the continuum limit approaches the time variable $t$ ):

$$
\theta_{m}^{n+1}=\mathbf{F}\left(\theta_{m}^{n-1}, \theta_{m+1}^{n}, \theta_{m-1}^{n}\right)
$$

and we assume that $\theta_{m}^{n}$ satisfies the periodic and even boundary conditions

$$
\theta_{m+M}^{n}=\theta_{m}^{n}, \quad \theta_{M-m}^{n}=\theta_{m}^{n}, \quad M=2 p, n \in \mathbb{Z}, m=0,1, \ldots, M-1, h=2 \pi / M
$$

Faddeev and Volkov [7] studied the symplectic structure of Hirota's equation on a spatially periodic lattice of even length $M=2 p$. We observe from (2.3) that $\theta_{m}^{n+1}$ only depends on $\theta_{m-1}^{n}, \theta_{m+1}^{n}$ and $\theta_{m}^{n-1}$, 


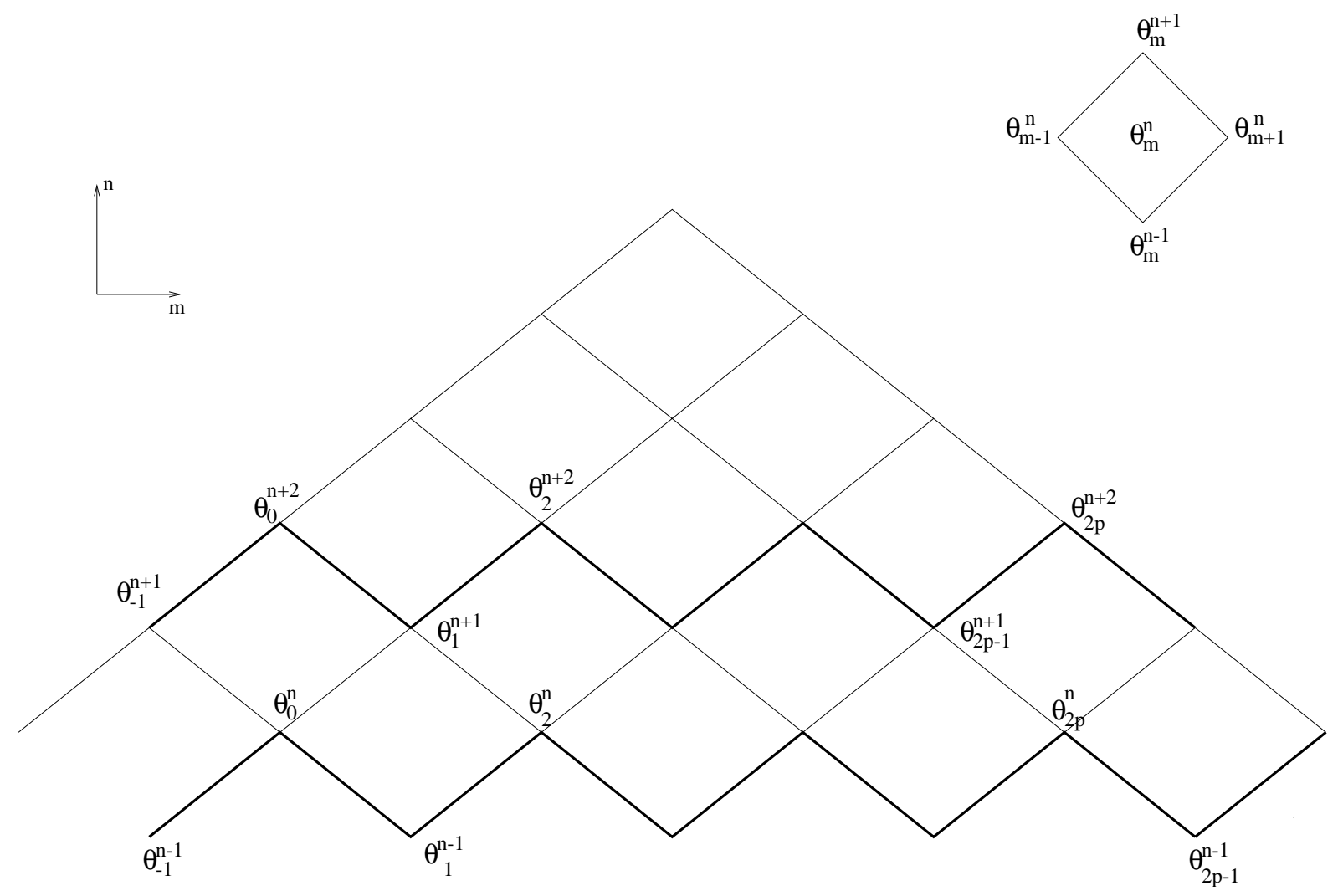

Figure 1: The geometry of double discrete SG equation.

there is no contribution from $\theta_{m}^{n}$. Assuming $M=2 p$, we specify initial values $\left\{\theta_{m}^{0}, m=1,3, \ldots, 2 p-1\right\}$ and $\left\{\theta_{m}^{n}, m=2,4, \ldots, 2 p\right\}$ and these values completely determine the solution for all $n \in \mathbb{Z}$. However, we may also consider the following initial values $\left\{\theta_{m}^{0}, m=2,4, \ldots, 2 p\right\}$ and $\left\{\theta_{m}^{1}, m=1,3, \ldots, 2 p-1\right\}$ which together with (2.3) again completely determine the solution for all times.

The phase space $\mathcal{L}$ of dDSG (2.3) is the $\mathbb{Z}^{2}$ with the interpretation that edges points in light cone directions. If we start with space periodic initial data $\theta_{j}^{i}$ along a saw $C_{n}$ on the light cone lattice (see Fig. 1) then the local evolution given by (2.3) (or (2.4)) will determine the function $\theta: \mathcal{L} \rightarrow \mathbb{R}$ on the whole lattice. In the smooth case the SG model describes the angle between the asymptotic line on an asymptotically parametrised $K$-surface. Bobenko and Pinkal [4] studied the geometric properties of the discrete $K$-surfaces (maps $\mathbb{Z}^{2} \rightarrow \mathbb{R}^{3}$ ) and proved that these surfaces parametrized by the second-order difference equation

$$
\theta_{m}^{n+1}+\theta_{m}^{n-1}=\theta_{m+1}^{n}+\theta_{m-1}^{n}+2 \mathrm{i} \operatorname{Arg}\left\{1+\frac{h^{2}}{4} \exp \left[\mathrm{i} \theta_{m+1}^{n}\right]\right\}++2 \mathrm{i} \operatorname{Arg}\left\{1+\frac{h^{2}}{4} \exp \left[\mathrm{i} \theta_{m-1}^{n}\right]\right\}
$$

which is equivelant to the Hirota equation (2.3).

In this paper, we consider the perturbed dDSG equation:

$$
\begin{aligned}
\theta_{m}^{n+1}+\theta_{m}^{n-1} & =\theta_{m+1}^{n}+\theta_{m-1}^{n}+2 \mathrm{i} \ln \left\{\frac{1+\frac{h^{2}}{4} \exp \left[\frac{\mathrm{i}}{2}\left(\theta_{m+1}^{n}+\theta_{m-1}^{n}\right)\right]}{1+\frac{h^{2}}{4} \exp \left[\frac{-\mathrm{i}}{2}\left(\theta_{m+1}^{n}+\theta_{m-1}^{n}\right)\right]}\right\} \\
& +\varepsilon\left\{\mathrm{b} \theta_{m}^{n}+\mathrm{c} \frac{\theta_{m+1}^{n}-2 \theta_{m}^{n}+\theta_{m-1}^{n}}{h^{2}}\right\}
\end{aligned}
$$


where $\theta_{m}^{n}$ satisfy the periodic boundary conditions (2.6) and $0<\varepsilon \ll 1, \mathrm{~b} \in\left(0, \mathrm{~b}_{0}\right), \mathrm{c} \in\left(0, \mathrm{c}_{0}\right), h=\frac{2 \pi}{M}<1$. When $\varepsilon=0$, the unperturbed dDSG is a completely integrable lattice equation. It has a spatially uniform solution $\hat{\theta}_{m}^{n}=\pi$.

We study the homoclinic structure for the dDSG equation and derive an analytic expression of the homoclinic orbits through the Bäcklund transformation and its Lax pair formulation. The homoclinic orbits approach the hyperbolic fixed point $\theta_{m}^{n}=\pi$ as $n \rightarrow \pm \infty$.

The system (2.8) is the (classical) double discrete model of the following near-integrable SG equation:

$$
\mathrm{u}_{t t}-\mathrm{u}_{x x}=\sin \mathrm{u}+\varepsilon\left(\mathrm{bu}+\mathrm{cu}_{x x}\right)
$$

when the lattice spacing $h$ tends to zero and the variables $\theta$ turn to the smooth function $\mathrm{u}(x, t)$, with $\mathrm{u}(x+2 \pi, t)=\mathrm{u}(x, t)=\mathrm{u}(-x, t)$ and $\varepsilon \in\left(0, \varepsilon_{1}\right), b, c>0$, (c.f. [2, 15, 21]). Thus, system (2.8) is of interest both as a perturbation of completely integrable lattice equation, finite dimension, and as an approximation to the PDE (2.9).

We shall prove the persistence of homoclinic orbits for the system (2.8). The Mel'nikov method [17] is one of the techniques that has proved to be very useful in dynamical systems. The method establishes a way to measure the distance between the stable and unstable manifolds of a saddle-type invariant set for a perturbed integrable system that originally has homoclinic structure.

\section{$3 \quad$ Integrability and Homoclinic Orbits}

In this section, we present two results concerned with homoclinic structure of dDSG equation. First, we construct the analytic expression of homoclinic orbits based on Bäcklund transformations of special solutions of dDSG. Then, we obtain an explicitly formula for the gradient of an important invariant of motion which we will use to build the distance function between the invariant manifolds.

\subsection{Integrable Background}

The dDSG equation that we want to study is the following nonlinear $\mathrm{P} \Delta \mathrm{E}$ :

$$
\sin \frac{1}{4}\left(\theta_{m}^{n}+\theta_{m+1}^{n+1}+\theta_{m}^{n+1}+\theta_{m+1}^{n}\right)=\frac{\mathrm{p}}{\mathrm{q}} \sin \frac{1}{4}\left(\theta_{m}^{n}+\theta_{m+1}^{n+1}-\theta_{m}^{n+1}-\theta_{m+1}^{n}\right)
$$

with boundary conditions (2.6). We note that $\theta_{m}^{n}$ is the dynamical variable at site $(n, m), n, m \in \mathbb{Z}$, and $\mathrm{p}, \mathrm{q} \in \mathbb{C}$ are lattice parameters. Equation (3.1) consists an alternative form of (2.2) or (2.3).

First we note that the dDSG (3.1) arises from a discrete action principle. The action for the dDSG reads $[5]$ :

$$
\begin{aligned}
S & =\sum_{n \in \mathbb{Z}} \sum_{m=0}^{M-1}\left\{\theta_{m+1}^{n}\left(\theta_{m+1}^{n+1}+\theta_{m}^{n}\right)-\frac{1}{4} \mathrm{~F}\left(2 \mathrm{i}(-1)^{m}\left(\theta_{m}^{n}+\theta_{m+1}^{n+1}\right)+\sigma_{2}\right)\right. \\
& \left.+\frac{1}{4} \mathrm{~F}\left(2 \mathrm{i}(-1)^{m}\left(\theta_{m}^{n}+\theta_{m+1}^{n+1}\right)+\sigma_{1}\right)\right\} \\
& \equiv \sum_{n \in \mathbb{Z}} \sum_{m=0}^{M-1} \mathrm{~L}\left(\theta_{m}^{n}, \theta_{m+1}^{n}, \theta_{m+1}^{n+1}, \theta_{m}^{n+1}\right)
\end{aligned}
$$

in which the function $\mathrm{F}$ is related to the dilogarithm function

$$
\mathrm{F}(x)=\int_{-\infty}^{x} \ln \left(1+\mathrm{e}^{\mathrm{i} \xi}\right) \mathrm{d} \xi
$$


and where $\sigma_{1}, \sigma_{2}$ are parameters related to the lattice parameters p, q:

$$
\mathrm{e}^{\sigma_{1}}=\frac{\mathrm{q}}{\mathrm{p}}, \quad \mathrm{e}^{\sigma_{2}}=\frac{\mathrm{p}}{\mathrm{q}}
$$

The Euler-Lagrange equation for (3.2) which are obtained by variation of $S$ with respect to th variables $\theta_{m}^{n}$, i.e

$$
\frac{\delta S}{\delta \theta_{m}^{n}}=0
$$

leads to the equation:

$$
\begin{aligned}
\theta_{m}^{n+1}+\theta_{m+1}^{n-1}+\theta_{m+1}^{n}+\theta_{m-1}^{n} & +\frac{\mathrm{i}(-1)^{m}}{2} \ln \left\{\frac{1+\exp \left[\sigma_{1}+2 \mathrm{i}(-1)^{m}\left(\theta_{m}^{n}+\theta_{m+1}^{n+1}\right)\right]}{1+\exp \left[\sigma_{2}+2 \mathrm{i}(-1)^{m}\left(\theta_{m}^{n}+\theta_{m+1}^{n+1}\right)\right]}\right\} \\
& +\frac{\mathrm{i}(-1)^{m}}{2} \ln \left\{\frac{1+\exp \left[\sigma_{2}+2 \mathrm{i}(-1)^{m}\left(\theta_{m}^{n}+\theta_{m-1}^{n-1}\right)\right]}{1+\exp \left[\sigma_{1}+2 \mathrm{i}(-1)^{m}\left(\theta_{m}^{n}+\theta_{m-1}^{n-1}\right)\right]}\right\} \\
& =0
\end{aligned}
$$

which is a consequence of equation (3.1).

Another important aspect of the dDSG, directly related to the integrability of this system, is that arises as the compatibility condition of an overdetermined linear system, Lax pair $[19,20]$ :

$$
\begin{gathered}
\phi_{m+1}^{n}(k)=\mathrm{V}_{n, m}(k) \phi_{m}^{n} \\
\phi_{m}^{n+1}(k)=\mathrm{U}_{n, m}(k) \phi_{m}^{n}
\end{gathered}
$$

where $k$ denotes the spectral parameter and $\mathrm{V}_{n, m}$ is given by

$$
\mathrm{V}_{n, m}(k)=\left(\begin{array}{cc}
\frac{\mathrm{q}}{\mathrm{q}-k} & \frac{v_{m+1}^{n}}{\mathrm{q}-k} \\
\frac{k^{2}}{\mathrm{q}-k} \frac{1}{v_{m}^{n}} & \frac{q}{\mathrm{q}-k} \frac{v_{m+1}^{n}}{v_{m}^{n}}
\end{array}\right), \quad v_{m}^{n}=\exp \left[2 \mathrm{i} \theta_{m}^{n}\right]
$$

and where $\mathrm{U}_{n, m}$ is given by a similar matrix obtained from (3.5) by making the replace $\mathrm{q} \rightarrow \mathrm{p}$ and $(n, m+1) \rightarrow(n+1, m)$.

Let $\mathrm{M}_{n, m}\left(k, \theta_{m}^{n}\right)$ be the fundamental matrix to the Lax pair, i.e the matrix solution to $(3.4 \mathrm{a}, 3.4 \mathrm{~b})$ with initial conditions $\mathrm{M}_{0}$ being a $2 \times 2$ identity matrix. The characteristic polynomial

$$
\operatorname{det}\left(\mathrm{M}_{n, m}\left(k, \theta_{m}^{n}\right)-\lambda \mathrm{I}\right)=0
$$

defines an invariant algebraic curve. The substitution

$$
\mathcal{M}=\lambda-\frac{\operatorname{traceM}_{n, m}(k)}{2}
$$

reduces this curve to the hyperelliptic form [4]:

$$
\mathcal{M}^{2}=\left(\frac{\operatorname{traceM}_{n, m}(k)}{2}\right)^{2}-\operatorname{det} \mathrm{M}_{n, m}(k)
$$

We define the function $\Delta$ as:

$$
\Delta: \mathbb{C} \times \mathcal{L} \longrightarrow \mathbb{C}
$$




$$
\Delta\left(k, \theta_{m}^{n}\right)=\operatorname{traceM}_{n, m}\left(M ; k, \theta_{m}^{n}\right)
$$

The spectral parameter $k \in \mathbb{C}$ fulfils the following condition

$$
-2 \mathrm{D} \leq \Delta\left(k, \theta_{m}^{n}\right) \leq 2 \mathrm{D}
$$

with $\mathrm{D}$ is constant and a critical point $k_{c}$ of $\Delta$ satisfies

$$
\left.\frac{\mathrm{d} \Delta}{\mathrm{d} k}\right|_{\left(k_{c}, \theta_{m}^{n}\right)}=0
$$

An important sequence of constant of motion $\mathcal{F}_{j}: \mathcal{L} \rightarrow \mathbb{C}$ is defined by

$$
\mathcal{F}_{j}\left(\theta_{m}^{n}\right)=\frac{1}{\mathrm{D}} \Delta\left(k_{c, j} ; \theta_{m}^{n}\right)
$$

\subsection{Analytic expression of homoclinic orbits}

We construct the homoclinic orbits for the dDSG through the Bäcklund transformation. For more complete studies on Bäcklund transformation see the book [22]. The unperturbed dDSG equation has an unstable hyperbolic fixed point at $\hat{\theta}_{m}^{n}=\pi$. We utilize the Bäcklund transformation for a concrete example, to generate homoclinic orbits through an iteration of the transformation to incorporate all the unstable and stable modes. The homoclinic orbits for the pde SG has been constructed by Ercolani et al. [6] through the Bäcklund transformation of fixed solutions.

Let $\phi_{m}^{n}$ denote a fix solution of the Lax pair at $\left(\theta_{m}^{n}, k_{d}\right)$, where $k_{d}$ is the double critical point of the function $\Delta$, i.e satisfies the condition (3.10) and $\Delta\left(k_{d} ; \theta_{m}^{n}\right)= \pm 2 \mathrm{D}$. We define the $2 \times 2$ transformation matrix $\Gamma_{n, m}$ by

$$
\Gamma_{n, m}=\left(\begin{array}{cc}
-\bar{k}-\alpha_{n, m} & \beta_{n, m} \\
\gamma_{n, m} & -\bar{k}-\delta_{n, m}
\end{array}\right)
$$

where

$$
\begin{gathered}
\alpha_{n, m}=\frac{k}{\mathbb{D}}\left(\left|\phi_{m}^{n, 1}\right|^{2}+\bar{k}^{2}\left|\phi_{m}^{n, 2}\right|^{2}\right), \quad \delta_{n, m}=\bar{\alpha}_{n, m} \\
\beta_{n, m}=\frac{\phi_{m}^{n, 1} \bar{\phi}_{m}^{n, 2}}{\mathbb{D}}\left(\bar{k}^{2}-|k|^{2}\right), \quad \gamma_{n, m}=-\bar{\beta}_{n, m}, \quad \mathbb{D}=\left|\phi_{m}^{n, 1}\right|^{2}+|k|^{2}\left|\phi_{m}^{n, 2}\right|^{2}
\end{gathered}
$$

We define

$$
\mathcal{V}_{m}^{n}=-\mathrm{p}\left(\bar{k}+\alpha_{n-1, m}+\left(1-\frac{k^{2}}{\mathrm{p}^{2}}\right)+\frac{\mathrm{p}-k}{v_{m}^{n}} \beta_{n, m}\right)\left(\bar{\beta}_{n-1, m}-\frac{\mathrm{p}-k}{\mathrm{p}} \frac{\bar{k}+\bar{\alpha}_{n-1, m}}{v_{m}^{n-1}}\right)^{-1}
$$

Then

$$
\Theta_{m}^{n}=\pi+\frac{1}{2 \mathrm{i}} \ln \mathcal{V}_{m}^{n}, \quad v_{m}^{n}=\mathrm{e}^{2 \mathrm{i} \theta_{m}^{n}}
$$

and

$$
\Phi_{m}^{n}(k)=\Gamma_{n, m}\left(k_{d}\right) \phi_{m}^{n}(k)
$$

Proposition 1 Let $\theta_{m}^{n}$ denote a solution of dDSG with double critical point $k_{d}$. We denote $\phi_{m}^{n}$ the general solution of the Lax pair at $\left(\theta_{m}^{n}, k_{d}\right)$. We define $\Theta_{m}^{n}$ and $\Phi_{m}^{n}(k)$ by (3.13) and (3.14).

i. $\Theta_{m}^{n}$ is also a solution of DDSG,

ii. $\Phi_{m}^{n}(k)$ solves the Lax pair (3.4a, 3.4b) at $\left(\Theta_{m}^{n}, k\right)$, 
iii. $\Theta_{m}^{n}$ is homoclinic to fixed solution $\hat{\theta}_{m}^{n}=\pi$, such that $\lim _{n \rightarrow \pm \infty} \Theta_{m}^{n}=\pi$.

Proof. We show that $\Phi_{m}^{n}(k)$ solves the Lax pair at $\left(\Theta_{m}^{n}, k\right)$ provided that $\phi_{m}^{n}$ solves $(3.4 \mathrm{a}, 3.4 \mathrm{~b})$ at $\left(\theta_{m}^{n}, k\right)$. From the equations of Lax pair we get

$$
\begin{aligned}
\Phi_{m+1}^{n}-\mathrm{V}_{n, m}\left(\Theta_{m}^{n}\right) \Phi_{m}^{n} & =\left[\Gamma_{n, m+1} \mathrm{~V}_{n, m}\left(\theta_{m}^{n}\right)-\mathrm{V}_{n, m}\left(\Theta_{m}^{n}\right) \Gamma_{n, m}\right] \Phi_{m}^{n} \\
\Phi_{m}^{n+1}-\mathrm{U}_{n, m}\left(\Theta_{m}^{n}\right) \Phi_{m}^{n} & =\left[\Gamma_{n+1, m} \mathrm{U}_{n, m}\left(\theta_{m}^{n}\right)-\mathrm{U}_{n, m}\left(\Theta_{m}^{n}\right) \Gamma_{n, m}\right] \Phi_{m}^{n}
\end{aligned}
$$

Hence if

$$
\begin{aligned}
& \Gamma_{n, m+1} \mathrm{~V}_{n, m}\left(\theta_{m}^{n}\right)-\mathrm{V}_{n, m}\left(\Theta_{m}^{n}\right) \Gamma_{n, m}=0 \\
& \Gamma_{n+1, m} \mathrm{U}_{n, m}\left(\theta_{m}^{n}\right)-\mathrm{U}_{n, m}\left(\Theta_{m}^{n}\right) \Gamma_{n, m}=0
\end{aligned}
$$

the proposition is established. In the component form equations $(3.15 \mathrm{a}, 3.15 \mathrm{~b})$ together the definitions (3.11-3.13) are equivalent to the eight algebraic equations. Indeed, the system

$$
\begin{aligned}
& \Gamma_{n, m+1}=\mathrm{V}_{n, m}\left(\Theta_{m}^{n}\right) \Gamma_{n, m} \mathrm{~V}_{n, m}^{-1}\left(\theta_{m}^{n}\right) \\
& \Gamma_{n+1, m}=\mathrm{U}_{n, m}\left(\Theta_{m}^{n}\right) \Gamma_{n, m} \mathrm{U}_{n, m}^{-1}\left(\theta_{m}^{n}\right)
\end{aligned}
$$

yields the following set of algebraic equations:

$$
\begin{gathered}
\mathcal{P}\left(\bar{k}+\alpha_{n+1, m}\right)=\left(\bar{k}+\alpha_{n, m}+\frac{\mathrm{p}-k}{\mathrm{p}} \frac{1}{v_{m}^{n+1}} \beta_{n, m}\right)-\frac{\mathcal{V}_{m}^{n+1}}{\mathrm{p}}\left(\gamma_{n, m}+(\mathrm{p}-k) \frac{\left(\bar{k}+\delta_{n, m}\right)}{\mathrm{p} v_{m}^{n+1}}\right) \\
\mathcal{P} \beta_{n+1, m}=\left(\bar{k}+\alpha_{n, m}\right) \frac{v_{m}^{n}}{\mathrm{p}}+\beta_{n, m} \frac{v_{m}^{n}}{v_{m}^{n+1}}-\frac{\mathcal{V}_{m}^{n+1}}{\mathrm{p}}\left(\bar{\beta}_{n, m} \frac{v_{m}^{n}}{\mathrm{p}}+\left(\bar{k}+\bar{\alpha}_{n, m}\right) \frac{v_{m}^{n}}{v_{m}^{n+1}}\right) \\
\mathcal{P} \gamma_{n+1, m}=-\frac{k^{2}}{\mathrm{p}} \frac{1}{\mathcal{V}_{m}^{n}}\left(\bar{k}+\alpha_{n, m}+\frac{\beta_{n, m}}{v_{m}^{n+1}} \frac{\mathrm{p}}{\mathrm{p}-k}\right)+\frac{\mathcal{V}_{m}^{n+1}}{\mathcal{V}_{m}^{n}}\left(\gamma_{n, m}+\frac{\bar{k}+\delta_{n, m}}{v^{n, m}} \frac{\mathrm{p}-k}{\mathrm{p}}\right) \\
\mathcal{P}\left(\bar{k}+\delta_{n+1, m}\right)=-\frac{k^{2}}{\mathrm{p} \mathcal{V}_{m}^{n}}\left(\left(\bar{k}+\alpha_{n, m}\right) \frac{v_{m}^{n}}{\mathrm{p}}+\beta_{n, m} \frac{v_{m}^{n}}{v_{m}^{n+1}}\right)+\frac{\mathcal{V}_{m}^{n+1}}{\mathcal{V}_{m}^{n}}\left(\gamma_{n, m} \frac{v_{m}^{n}}{\mathrm{p}}+\left(\bar{k}+\delta_{n, m}\right) \frac{v_{m}^{n}}{v_{m}^{n+1}}\right) \\
\mathcal{Q}\left(\bar{k}+\alpha_{n, m+1}\right)=\left(\bar{k}+\alpha_{n, m}+\frac{\mathrm{q}-k}{\mathrm{q}} \frac{\beta_{n, m+1}}{v_{m}^{n+1}}\right)-\frac{\mathcal{V}_{m+1}^{n}}{\mathrm{q}}\left(\gamma_{n, m}+\frac{\mathrm{q}-k}{\mathrm{q}} \frac{\bar{k}+\delta_{n, m}}{v_{m+1}^{n}}\right) \\
\mathcal{Q} \beta_{n, m+1}=\left(\left(\bar{k}+\alpha_{n, m}\right) \frac{v_{m}^{n}}{\mathrm{q}}+\beta_{n, m} \frac{v_{m}^{n}}{v_{m+1}^{n}}\right)-\frac{\mathcal{V}_{m+1}^{n}}{\mathrm{q}}\left(\gamma_{n, m} \frac{v_{m}^{n}}{\mathrm{q}}+\left(\bar{k}+\delta_{n, m}\right) \frac{v_{m}^{n}}{v_{m+1}^{n}}\right) \\
\mathcal{Q} \gamma_{n, m+1}=-\frac{k^{2}}{\mathrm{q} \mathcal{V}_{m}^{n}}\left(\bar{k}+\alpha_{n, m}+\frac{\mathrm{q}-k}{\mathrm{q}} \frac{\beta_{n, m}}{v_{m+1}^{n}}\right)+\frac{\mathcal{V}_{m+1}^{n}}{\mathcal{V}_{m}^{n}}\left(\gamma_{n, m}+\frac{\mathrm{q}-k}{\mathrm{q}+\delta_{n, m}} \frac{\bar{v}_{m+1}^{n}}{\mathrm{q}}\right) \\
\mathcal{Q}\left(\bar{k}+\delta_{n, m+1}\right)=-\frac{k^{2}}{\mathrm{q} \mathcal{V}_{m}^{n}}\left(\left(\bar{k}+\alpha_{n, m}\right) \frac{v_{m}^{n}}{\mathrm{q}}+\frac{v_{m}^{n}}{v_{m+1}^{n}} \beta_{n, m}\right)+\frac{\mathcal{V}_{m}^{n+1}}{\mathcal{V}_{m}^{n}}\left(\gamma_{n, m} \frac{v_{m}^{n}}{\mathrm{q}}+\left(\bar{k}+\delta_{n, m}\right) \frac{v_{m}^{n}}{v_{m+1}^{n}}\right)
\end{gathered}
$$

where

$$
\mathcal{P}=\left(1-\frac{k^{2}}{\mathrm{p}^{2}}\right)^{-1}, \quad \mathcal{Q}=\left(1-\frac{k^{2}}{\mathrm{q}^{2}}\right)^{-1}
$$

The problem is reduced to checking these eight equations. From representation of $\alpha_{n . m}, \beta_{n, m}, \gamma_{n, m}, \delta_{n, m}$ and $\mathcal{V}_{m}^{n}$ this is done explicitly.

As mentioned, $\hat{\theta}_{m}^{n}=\pi$ is a solution of the dDSG with unstable direction. In this case the potential function on the Lax pair becomes $v_{m}^{n}=1$, thus the solutions of $(3.4 \mathrm{a}, 3.4 \mathrm{~b})$ takes the form:

$$
\phi_{m}^{n(-)}=(1,-k)^{\perp} \lambda_{-}^{m}, \quad \phi_{m}^{n(+)}=(1, k)^{\perp} b^{n} \lambda_{+}^{m}
$$


where

$$
\lambda_{ \pm}=a \pm \frac{k a}{\mathrm{q}}, \quad a:=\frac{\mathrm{q}}{\mathrm{q}-k}, \quad b:=\frac{\mathrm{p}+k}{\mathrm{p}-k}
$$

and $k=k_{d}$ is a double critical point of $\Delta$ this means that satisfies the conditions:

$$
\Delta(k)=2 \mathrm{D}, \quad \frac{\mathrm{d}}{\mathrm{d} k} \Delta(k)=0
$$

with

$$
\Delta(k)=\left(a-\frac{k a}{\mathrm{q}}\right)^{m}+b^{n} k\left(a+\frac{k a}{\mathrm{q}}\right)^{m}, \quad\left|M_{n, m}\right|:=2 b^{n} k\left(a^{2}-\left(\frac{k a}{\mathrm{q}}\right)^{2}\right)^{m}
$$

and

$$
\operatorname{det}_{n, M}\left(\phi^{-}, \phi^{+}\right)=\mathrm{D}^{2} \operatorname{det} \mathrm{M}_{n, 0}, \quad \text { with } \quad \mathrm{D}^{2}:=\prod_{m=0}^{M-1}\left(\lambda_{-} \lambda_{+}\right)^{m}
$$

Insertion $(3.17)$ to $(3.12,3.13)$ and making use of the identity $\tan ^{-1} X=\frac{1}{2 \mathrm{i}} \ln \left[\frac{1+\mathrm{i} X}{1-\mathrm{i} X}\right]$, we obtain the following analytic expression of homoclinic orbits:

$$
\Theta_{m}^{n}=\pi+4 \tan ^{-1}\left(\mathrm{P} \cos \left(m h \mathrm{w}_{1}+\mathrm{x}\right) \operatorname{sech}\left(n h \mathrm{w}_{2}+\tau\right)\right)
$$

with

$$
\mathrm{P}^{2}=\frac{\sinh ^{2} \mathrm{w}_{2} h}{\sin ^{2} \mathrm{w}_{1} h} \quad \text { and } \quad\left(1-\frac{h^{2}}{4}\right) \cosh _{2} h=\left(1+\frac{h^{2}}{4}\right) \cos _{1} h, \quad h=2 \pi / M
$$

and $\lim _{n \rightarrow \pm \infty} \Theta_{m}^{n}=\pi$

Remark 1 The expression of homoclinic orbits (3.20) with the conditions (3.21) satisfies the dDSG equation. We rewrite the homoclinic solutions as follows:

$$
\Theta_{m}^{n}=4 \tan ^{-1}\left(\mathrm{~A}_{m}^{n}\right), \quad \mathrm{A}_{m}^{n}=\mathrm{P} \cos \left(m h \mathrm{w}_{1}+\mathrm{x}\right) \operatorname{sech}\left(n h \mathrm{w}_{2}+\tau\right)
$$

Substitution of the solutions (3.22) to (2.2) and the identity $\tan ^{-1} X=\frac{1}{2 \mathrm{i}} \ln \left[\frac{1+\mathrm{i} X}{1-\mathrm{i} X}\right]$ yield an equation of the form

$$
\left[1-\frac{h^{2}}{4}\right] \frac{1-\mathrm{A}_{m+1}^{n} \mathrm{~A}_{m-1}^{n}}{\mathrm{~A}_{m+1}^{n}+\mathrm{A}_{m-1}^{n}}=\left[1+\frac{h^{2}}{4}\right] \frac{1-\mathrm{A}_{m}^{n+1} \mathrm{~A}_{m}^{n-1}}{\mathrm{~A}_{m}^{n+1}+\mathrm{A}_{m}^{n-1}}
$$

Using the conditions (3.21) and after some manipulations we obtain the desired identity.

Remark 2 The continuum limit of the discrete homoclinic orbits (3.20) coincides with the homoclinic orbits of the field $S G,[6,21]$.

\subsection{The gradient of $\Delta\left(k, \theta_{m}^{n}\right)$}

The trace of the fundamental matrix of Lax pair is an important invariant of motion for the sine-Gordon equation. The corresponding invariants $\mathcal{F}_{j}\left(\theta_{m}^{n}\right)$ for the dDSG system are defined as:

$$
\mathcal{F}_{j}\left(\theta_{m}^{n}\right)=\frac{1}{\mathrm{D}} \Delta\left(k_{j}^{c}\left(\theta_{m}^{n}\right), \theta_{m}^{n}, \theta_{m+1}^{n}\right), \quad v_{m}^{n}=\mathrm{e}^{2 \mathrm{i} \theta_{m}^{n}}
$$

We shall use the invariants $\mathcal{F}_{j}$ to build the Mel'nikov functions, in Section 4. 
Lemma 1 Let $k_{j}^{c}\left(\theta_{m}^{n}, \theta_{m+1}^{n}\right)$ be a simple critical point of $\Delta$;

$$
\begin{aligned}
\frac{\delta \mathcal{F}_{j}}{\delta \theta_{m}^{n}}\left(\theta_{m}^{n}, \theta_{m+1}^{n}\right) & =\frac{1}{\mathrm{D}} \frac{\delta \Delta}{\delta \theta_{m}^{n}}\left(k_{j}^{c}\left(\theta_{m}^{n}\right), \theta_{m}^{n}, \theta_{m+1}^{n}\right) \\
\frac{\delta \mathcal{F}_{j}}{\delta \theta_{m+1}^{n}}\left(\theta_{m}^{n}, \theta_{m+1}^{n}\right) & =\frac{1}{\mathrm{D}} \frac{\delta \Delta}{\delta \theta_{m+1}^{n}}\left(k_{j}^{c}\left(\theta_{m}^{n}\right), \theta_{m}^{n}, \theta_{m+1}^{n}\right)
\end{aligned}
$$

where

$$
\begin{aligned}
\frac{\delta \Delta}{\delta \theta_{m}^{n}}\left(k, \theta_{m}^{n}\right) & =\frac{2 \mathrm{i}}{\mathrm{q}-k} \operatorname{trace}\left[\mathrm{M}_{n, m+1}^{-1}\left(\begin{array}{cc}
0 & 0 \\
\frac{k^{2}}{v_{m}^{n}} & -\mathrm{q} \frac{v_{m+1}^{n}}{v_{m}^{n}}
\end{array}\right) \mathrm{M}_{n, m} \mathrm{M}_{n, M}\right] \\
\frac{\delta \Delta}{\delta \theta_{m+1}^{n}}\left(k, \theta_{m}^{n}\right) & =\frac{2 \mathrm{i}}{\mathrm{q}-k} \operatorname{trace}\left[\mathrm{M}_{n, m+1}^{-1}\left(\begin{array}{cc}
0 & v_{m+1}^{n} \\
0 & \mathrm{q} \frac{v_{m+1}^{n}}{v_{m}^{n}}
\end{array}\right) \mathrm{M}_{n, m} \mathrm{M}_{n, M}\right]
\end{aligned}
$$

$v_{m}^{n}=\mathrm{e}^{2 \mathrm{i} \theta_{m}^{n}}$ and $\mathrm{M}_{n, m}=\left[\phi_{m}^{n(-)}, \phi_{m}^{n(+)}\right], \phi_{m}^{n,( \pm)}=\left(\phi_{m}^{n( \pm, 1)}, \phi_{m}^{n( \pm, 2)}\right)$ are two Bloch functions of the Lax pair (3.4a, 3.4b) and $\left|\mathrm{M}_{n, m}\right|=\operatorname{det} \mathrm{M}_{n, m}$.

Proof. Since $k_{j}^{c}$ be a critical point, $\Delta^{\prime}\left(k_{j}^{c}\left(\theta_{m}^{n}\right), \theta_{m}^{n}\right)=0$ and

$$
\frac{\delta k_{j}^{c}}{\delta \theta_{m}^{n}}=-\frac{1}{\Delta^{\prime \prime}} \frac{\delta \Delta^{\prime}}{\delta \theta_{m}^{n}}
$$

then $k_{j}^{c}$ is a differential function

$$
\mathrm{D} \frac{\delta \mathcal{F}_{j}}{\delta \theta_{m}^{n}}\left(k, \theta_{m}^{n}\right)=\left.\frac{\delta \Delta}{\delta \theta_{m}^{n}}\right|_{k=k_{j}^{c}}+\left.\frac{\delta \Delta}{\delta k}\right|_{k=k_{j}^{c}} \frac{\delta k_{j}^{c}}{\delta \theta_{m}^{n}}=\left.\frac{\delta \Delta}{\delta \theta_{m}^{n}}\right|_{k=k_{j}^{c}}
$$

Let $\mathrm{M}_{n, m}$ be the fundamental matrix of the Lax pair, for the special potential function $v_{m}^{n}=\mathrm{e}^{2 \mathrm{i} \theta_{m}^{n}}$. Variation of the $v_{m}^{n}$ leads to the variational equation for the variation of $\mathrm{M}_{n, m}$ at fixed $k$ :

$$
\begin{aligned}
\mathrm{M}_{n, m+1} & =\mathrm{V}_{n, m} \mathrm{M}_{n, m} \\
\delta \mathrm{M}_{n, m+1} & =\mathrm{V}_{n, m} \delta \mathrm{M}_{n, m}+\delta \mathrm{V}_{n, m} \mathrm{M}_{n, m} \\
\delta \mathrm{V}_{n, m} & =\left(\begin{array}{cc}
0 & \frac{1}{\mathrm{q}-k} \delta v_{m+1}^{n} \\
\frac{k^{2}}{\mathrm{q}-k} \delta\left(\frac{1}{v_{m}^{n}}\right) & \frac{\mathrm{q}}{\mathrm{q}-k} \delta\left(\frac{v_{m+1}^{n}}{v_{m}^{n}}\right)
\end{array}\right)
\end{aligned}
$$

Let $\delta \mathrm{M}_{n, m}=\mathrm{M}_{n, m} \mathrm{~A}_{n, m}$, where $\mathrm{A}_{n, m} 2 \times 2$ matrix to be determined.

$$
\begin{aligned}
\delta \mathrm{M}_{n, m+1} & =\mathrm{M}_{n, m+1} \mathrm{~A}_{n, m+1}=\mathrm{V}_{n, m} \mathrm{M}_{n, m} \mathrm{~A}_{n, m}+\delta \mathrm{V}_{n, m} \mathrm{M}_{n, m} \\
& =\mathrm{M}_{n, m+1} \mathrm{~A}_{n, m}+\delta \mathrm{V}_{n, m} \mathrm{M}_{n, m}
\end{aligned}
$$

thus,

$$
\mathrm{A}_{n, m+1}-\mathrm{A}_{n, m}=\mathrm{M}_{n, m+1}^{-1} \delta \mathrm{V}_{n, m} \mathrm{M}_{n, m}
$$




$$
\mathrm{A}_{n, 0}=0
$$

Solving the system (3.25) we have,

$$
\delta \mathrm{M}_{n, m}=\mathrm{M}_{n, M}\left[\sum_{\jmath=1}^{M-1} \mathrm{M}_{n, \jmath}^{-1} \delta \mathrm{V}_{n, \jmath-1} \mathrm{M}_{n, \jmath-1}\right], \quad \delta \mathrm{M}_{n, 0}=0
$$

Then,

$$
\delta \Delta\left(k, \theta_{m}^{n}\right)=\operatorname{trace}\left\{M_{n, M}\left[\sum_{\jmath=1}^{M-1} \mathrm{M}_{n, \jmath}^{-1} \delta \mathrm{V}_{n, \jmath-1} \mathrm{M}_{n, \jmath-1}\right]\right\}
$$

thus, we obtain the equations (3.24). Substitution the representation of $\mathrm{M}_{n, m}$ into (3.24) entails:

$$
\begin{aligned}
\frac{\delta \Delta}{\delta \theta_{m}^{n}}\left(k, \theta_{m}^{n}\right) & =\frac{2 \mathrm{i}}{\mathrm{q}-k} \frac{1}{\left|\mathrm{M}_{n, m+1}\right|}\left\{-\frac{k^{2}}{v_{m}^{n}}\left[\phi_{m+1}^{n(+1)}\left(\phi_{m}^{n(-1)} \phi_{M}^{n(-1)}+\phi_{m}^{n(+1)} \phi_{M}^{n(-2)}\right)\right.\right. \\
& \left.+\phi_{m+1}^{n(-2)}\left(\phi_{m}^{n(-1)} \phi_{M}^{n(+1)}+\phi_{m}^{n(+1)} \phi_{M}^{n(+2)}\right)\right]+\mathrm{q} \frac{v_{m+1}^{n}}{v_{m}^{n}}\left[\phi _ { m } ^ { n ( - 1 ) } \left(\phi_{m}^{n(-1)} \phi_{M}^{n(+1)}\right.\right. \\
& \left.\left.\left.+\phi_{m}^{n(+2)} \phi_{M}^{n(+2)}\right)-\phi_{m+1}^{n(+1)}\left(\phi_{m}^{n(-2)} \phi_{M}^{n(-1)}+\phi_{m}^{n(+2)} \phi_{M}^{n(-2)}\right)\right]\right\} \\
\frac{\delta \Delta}{\delta \theta_{m+1}^{n}}\left(k, \theta_{m}^{n}\right) & =\frac{2 \mathrm{i}}{\mathrm{q}-k} \frac{1}{\left|\mathrm{M}_{n, m+1}\right|}\left\{v _ { m + 1 } ^ { n } \left[\phi_{m}^{n(+2)}\left(\phi_{m}^{n(-2)} \phi_{M}^{n(-1)}+\phi_{m}^{n(+2)} \phi_{M}^{n(-2)}\right)\right.\right. \\
& \left.-\phi_{m}^{n(-2)}\left(\phi_{m}^{n(-2)} \phi_{M}^{n(+1)}+\phi_{m}^{n(+2)} \phi_{M}^{n(+2)}\right)\right]+\mathrm{q} \frac{v_{m+1}^{n}}{v_{m}^{n}}\left[\phi _ { m } ^ { n ( - 1 ) } \left(\phi_{m}^{n(-2)} \phi_{M}^{n(+1)}\right.\right. \\
& \left.\left.\left.+\phi_{m}^{n(+2)} \phi_{M}^{n(+2)}\right)-\phi_{m}^{n(+1)}\left(\phi_{m}^{n(-2)} \phi_{M}^{n(-1)}+\phi_{m}^{n(+2)} \phi_{M}^{n(-2)}\right)\right]\right\}
\end{aligned}
$$

Substitute $(3.17,3.20)$ into $(3.26 \mathrm{a}, 3.26 \mathrm{~b})$, we obtain the expressions of $\frac{\delta \Delta}{\delta \theta_{m}^{n}}$ evaluated on the homoclinic orbits. This completes the proof of the Lemma.

\section{Homoclinic Orbits for perturbed dDSG equation}

Next, we will establish the persistence of homoclinic orbits (3.20) for the perturbed dDSG equation (2.8). First, we show that the solution $\hat{\theta}_{m}^{n}=\pi$ is a singular fixed point of the integrable dDSG equation and second, we prove that, for sufficiently small $\varepsilon>0$, there exists a solution homoclinic to $\hat{\theta}_{m}^{n}(\varepsilon)$.

\subsection{Linearized analysis}

In this subsection, we discuss the linear stability analysis of the fixed solutions of dDSG (2.8). We note that if $\theta_{m}^{n}$ solves the equation (2.3) then $(-1)^{n+m} \theta_{m}^{n}$ is also a solution. Assume that the small perturbation $\hat{\theta}_{m}^{n}(\varepsilon)$ are exponentially fast growing, as a consequence of the linearly unstable modes of the continuum SG equation [21]

$$
\theta_{m}^{n}=\hat{\theta}_{m}^{n}(\varepsilon)+\eta \varphi_{m}^{n}, \quad 0<\eta \ll 1
$$

with

$$
\varphi_{m}^{n}=\Omega_{k}^{n} \exp \left[\mathrm{i} \mathcal{A}_{k} m\right], \quad \mathcal{A}_{k}=\frac{2 \pi k}{M}
$$


where $\hat{\theta}_{m}^{n}(\varepsilon)=\pi+\mathcal{O}(\varepsilon)$ is a solution of $(2.8), M=2 p$ the total number of grid points in the space interval $(0,1)$ and $k=-\frac{M}{2}, \ldots, \frac{M}{2}-1$.

Linearize the equation (2.8) we obtain

$$
\begin{aligned}
\frac{\varphi_{m}^{n+1}-2 \varphi_{m}^{n}+\varphi_{m}^{n-1}}{h^{2}} & -\frac{\varphi_{m+1}^{n}-2 \varphi_{m}^{n}+\varphi_{m-1}^{n}}{h^{2}}-\frac{1}{4}\left(\varphi_{m}^{n+1}+\varphi_{m}^{n-1}+\varphi_{m+1}^{n}+\varphi_{m-1}^{n}\right) \\
& =\varepsilon\left\{\mathrm{b} \varphi_{m}^{n}+\mathrm{c} \frac{\varphi_{m+1}^{n}-2 \varphi_{m}^{n}+\varphi_{m-1}^{n}}{h^{2}}\right\}
\end{aligned}
$$

After straightforward calculations, we take the following equation for eigenvalues $\Omega_{k}$ :

$$
\Omega_{k}^{2}-2\left(\frac{\cos \mathcal{A}_{k}}{\mathrm{a}}+\varepsilon\left(\frac{\mathrm{b} h^{2}}{2}-\mathrm{c} \sin ^{2} \frac{\mathcal{A}_{k}}{2}\right)\right) \Omega_{k}+1=0
$$

with

$$
\mathrm{a}=\left(1-\frac{h^{2}}{4}\right)\left(1+\frac{h^{2}}{4}\right)^{-1} \quad h=2 \pi / M
$$

and

$$
\Omega_{k}=\left(\frac{\cos \mathcal{A}_{k}}{\mathrm{a}}+\varepsilon\left(\frac{\mathrm{b} h^{2}}{2}-\mathrm{c} \sin ^{2} \frac{\mathcal{A}_{k}}{2}\right)\right) \pm \sqrt{\left|\left(\frac{\cos \mathcal{A}_{k}}{\mathrm{a}}+\varepsilon\left(\frac{\mathrm{b} h^{2}}{2}-\mathrm{c} \sin ^{2} \frac{\mathcal{A}_{k}}{2}\right)\right)^{2}-1\right|}
$$

The stability condition $\left|\Omega_{k}\right| \leq 1$ becomes:

$$
\left(\frac{\cos \mathcal{A}_{k}}{\mathrm{a}}+\varepsilon\left(\frac{\mathrm{b} h^{2}}{2}-\mathrm{c} \sin ^{2} \frac{\mathcal{A}_{k}}{2}\right)\right)^{2} \leq 1
$$

It follows after some manipulations that the dispersion relation at $\mathcal{A}_{k}=2 \pi k / M$ may be written as follows:

$$
\Omega_{k}=\frac{\cos \mathcal{A}_{k}}{\mathrm{a}}+\varepsilon\left(\frac{\mathrm{b} h^{2}}{2}-\operatorname{c\operatorname {sin}^{2}} \frac{\mathcal{A}_{k}}{2}\right) \pm 2 \mathrm{i} \cos ^{2} \frac{\mathcal{A}_{k}}{2} \sqrt{\mathrm{E}_{k} \mathrm{~F}_{k}}
$$

for $k=-\frac{M}{2}, \ldots, \frac{M}{2}-1, k \neq 0$ and

$$
\begin{aligned}
& \mathrm{E}_{k}:=\frac{h^{2}}{4}-\tan ^{2} \frac{\mathcal{A}_{k}}{2}+\varepsilon \frac{1-\frac{h^{2}}{4}}{2 \cos ^{2} \frac{\mathcal{A}_{k}}{2}}\left(\frac{\mathrm{b} h^{2}}{2}-\mathrm{c} \sin ^{2} \frac{\mathcal{A}_{k}}{2}\right)+\mathcal{O}\left(\varepsilon^{2}\right) \\
& \mathrm{F}_{k}:=1-\frac{h^{2}}{4} \tan ^{2} \frac{\mathcal{A}_{k}}{2}+\varepsilon \frac{1-\frac{h^{2}}{4}}{2 \cos ^{2} \frac{\mathcal{A}_{k}}{2}}\left(\frac{\mathrm{b} h^{2}}{2}-\mathrm{c} \sin ^{2} \frac{\mathcal{A}_{k}}{2}\right)+\mathcal{O}\left(\varepsilon^{2}\right)
\end{aligned}
$$

The stability condition (4.6) is violated by the wave number $\mathcal{A}_{k} \simeq 0$ for $k=0$. The unstable wave numbers satisfy:

$$
\left(\frac{\cos \mathcal{A}_{k}}{\mathrm{a}}+\varepsilon\left(\frac{\mathrm{b} h^{2}}{2}-\mathrm{c} \sin ^{2} \frac{\mathcal{A}_{k}}{2}\right)-1\right)\left(\frac{\cos \mathcal{A}_{k}}{\mathrm{a}}+\varepsilon\left(\frac{\mathrm{b} h^{2}}{2}-\mathrm{c} \sin ^{2} \frac{\mathcal{A}_{k}}{2}\right)+1\right)>0
$$

which implies the following:

$$
\tan ^{2} \frac{\mathcal{A}_{k}}{2}<\frac{h^{2}}{4}\left(1+\varepsilon\left(1-\frac{h^{2}}{4}\right)\left(\frac{\mathrm{b}}{\cos \frac{\mathcal{A}_{k}}{2}}-\frac{\mathrm{c}}{2}\right)\right)+\mathcal{O}\left(\varepsilon^{2}\right)
$$

For $k=0$, the perturbed fixed point $\hat{\theta}_{m}^{n}(\varepsilon)$ loses stability and is turned into an unstable hyperbolic fixed point with

$$
\Omega_{0}=\frac{1}{\mathrm{a}}+\varepsilon \frac{\mathrm{b} h^{2}}{2} \pm h \sqrt{\left(1+\varepsilon\left(1-\frac{h^{2}}{4}\right) \mathrm{b}\right)\left(1+\varepsilon\left(1-\frac{h^{2}}{4}\right) \frac{\mathrm{b} h^{2}}{4}\right)}
$$




\subsection{Mel'nikov method}

With the geometric structures constructed in the previous sections, we start to construct orbits for the perturbed dDSG (2.8) homoclinic to the saddle point $\hat{\theta}_{m}^{n}(\varepsilon)$. The construction is composed of two steps. We start with an initial point in the unstable manifold of $\hat{\theta}_{m}^{n}(\varepsilon)$, and first we show that the forward orbit enters the center-stable manifold of the neighborohood $\mathcal{M}_{\varepsilon}$, if the parameters b, c is properly chosen. Second, we prove that the perturbed orbits approach the fixed point $\hat{\theta}_{m}^{n}(\varepsilon)$ in forward time $n \rightarrow+\infty$. The Mel'nikov method is the main tool.

We write the perturbed dDSG equation (2.8) in the form:

$$
\theta_{m}^{n+1}=\mathbf{F}\left(\theta_{m}^{n-1}, \theta_{m \pm 1}^{n}\right)+\varepsilon \mathbf{G}\left(\theta_{m}^{n}, \theta_{m \pm 1}^{n}\right)
$$

G denotes the perturbation:

$$
\mathbf{G}:=\mathrm{b} \theta_{m}^{n}+\mathrm{c} \frac{\theta_{m+1}^{n}-2 \theta_{m}^{n}+\theta_{m-1}^{n}}{h^{2}}
$$

Before we discuss the persistent homoclinic orbits, we make some observations related to the existence of an invariant subspace for the dDSG. In particular, the dDSG equation admits an invariant subspace $\Pi$. Indeed, if we consider the constraint:

$$
\theta_{m+1}^{n}=\theta_{m-1}^{n}=\theta_{m}^{n}, \quad m=0,1, \ldots, M-1, M=2 p
$$

On the plane $\Pi$ the perturbed system has a saddle fixed point $\hat{\theta}_{m}^{n}(\varepsilon)$ and in the full phase space linear stability analysis shows that $\hat{\theta}_{m}^{n}(\varepsilon)$ is a saddle point and by the invariant manifold theory $W^{\mathrm{u}}\left(\hat{\theta}_{m}^{n}(\varepsilon)\right)$ exists and is two-dimensional (two unstable directions in and off the plane $\Pi$ ). $W^{\mathrm{s}}\left(\hat{\theta}_{m}^{n}(\varepsilon)\right.$ ) also exists and has codimension 2 and $W_{\varepsilon}^{\mathrm{cs}}$ has codimension 1 . The intersection $W^{\mathrm{u}}\left(\hat{\theta}_{m}^{n}(\varepsilon)\right) \cap W_{\varepsilon}^{\mathrm{cs}}$ will be onedimensional.

Thus, the unstable, stable and centre manifolds of the map $\mathbf{F}$ (c.f (2.5)) associated to $\hat{\theta}_{m}^{n}$ :

$$
\begin{aligned}
W^{\mathrm{u}} & =\left\{\theta_{m}^{n} \in \mathcal{L}: \lim _{n \rightarrow-\infty} \mathbf{F}^{n}\left(\theta_{m}^{n}\right)=\hat{\theta}_{m}^{n}\right\} \\
W^{\mathrm{s}} & =\left\{\theta_{m}^{n} \in \mathcal{L}: \lim _{n \rightarrow+\infty} \mathbf{F}^{n}\left(\theta_{m}^{n}\right)=\hat{\theta}_{m}^{n}\right\}
\end{aligned}
$$

$W^{\mathrm{s}}\left(\hat{\theta}_{m}^{n}(\varepsilon)\right) \subset W_{\varepsilon}^{\mathrm{cs}}$ and $\mathcal{M}_{\varepsilon}$ be a neighborhood such that the whole plane $\Pi$ lies inside $\mathcal{M}_{\varepsilon}$.

For the perturbed system, we have the local stable and unstable manifolds $W_{\varepsilon, \text { loc }}^{\mathrm{s}, \mathrm{u}}$ of the perturbed point $\hat{\theta}_{m}^{n}(\varepsilon)$ are " $\varepsilon$-close" to those of the unperturbed point $\hat{\theta}_{m}^{n}=\pi$.

Consider the saddle fixed point $\hat{\theta}_{m}^{n}(\varepsilon)$ on the invariant plane $\Pi$ and we seek an orbit, not on $\Pi$, which is homoclinic to $\hat{\theta}_{m}^{n}(\varepsilon)$. We present a geometrical mean to establish the persistence of homoclinic orbits for the near-integrable dDSG equation. Our method based on the Mel'nikov measurement through which we answer the question : Is there any intersection between $W_{\text {loc }}^{\mathrm{u}}\left(\hat{\theta}_{m}^{n}(\varepsilon)\right)$ and $W^{\mathrm{s}}\left(\mathcal{M}_{\varepsilon}\right) \subset W_{\varepsilon}^{\mathrm{cs}}$ ?

The homoclinic orbits of the dDSG equation approach the subspace

$$
\Pi=\left\{\theta_{m}^{n} \in \mathcal{L}: \theta_{m+1}^{n}=\theta_{m-1}^{n}=\theta_{m}^{n}, m=0,1, \ldots, M-1\right\}
$$

as $n \rightarrow+\infty$. Our analysis of the near-integrable dDSG equation begins with two observations: First, when $\varepsilon=0$, the lattice SG equation is a completely integrable system. Second, the subspace $\Pi$ is an invariant space for perturbed dDSG. In each of these two cases $\left(\varepsilon=0\right.$ or $\left.\theta_{m}^{n} \in \Pi\right)$ the behaviour of solution $\theta_{m}^{n}$ (as $n \in \mathbb{Z}$ ) can be described completely. In the first case, this description is described in Section 3, through the Bäcklund transformation of the solution $\pi$ and Lax pair, in the second case it is accomplished through phase plane analysis of the reduced map (discrete pendulum). In the jargon 
of the theory of dynamical systems our method will be a form of "local-global" analysis, where at times the term "local" will mean close to the subspace $\Pi$ of the discrete pendulum, and at other times "local" will mean close to the integrable solutions, homoclinic with respect to the index $n$. In any event, throughout the global arguments, Mel'nikov method, control is achieved either because of proximity to (i) the subspace $\Pi$ or (ii) $\varepsilon=0$.

The essence of Mel'nikov method is as follows: Firstly, we establish the intersection of $W_{\varepsilon}^{\mathrm{cs}}$ and $W_{\varepsilon, \text { loc }}^{\mathrm{u}}\left(\hat{\theta}_{m}^{n}(\varepsilon)\right)$ of the perturbed dDSG equation, so that for external parameters in a fixed open set, there are orbits which tend to $\hat{\theta}_{m}^{n}(\varepsilon)$ when $n \rightarrow-\infty$ and approach $\Pi$ when $n \rightarrow+\infty$. Secondly, we prove that these orbits also tend to $\hat{\theta}_{m}^{n}(\varepsilon)$ in forward "time" $(n \rightarrow+\infty)$.

Let $\mathrm{h}_{m}^{n}$ be the homoclinic orbits of the dDSG and $\mathrm{h}_{m}^{n, \mathrm{u}}(\varepsilon), \mathrm{h}_{m}^{n, \mathrm{~s}}(\varepsilon)$ orbits lie on $W_{\varepsilon, \text { loc }}^{\mathrm{u}}\left(\hat{\theta}_{m}^{n}(\varepsilon)\right)$ and $W_{\varepsilon, \text { loc }}^{\mathrm{s}}\left(\hat{\theta}_{m}^{n}(\varepsilon)\right)$ respectively $\varepsilon$-close to $\mathrm{h}_{m}^{n}$. We give the following parametrization for these orbits

$$
\begin{gathered}
\mathrm{h}_{m}^{n, \mathrm{u}}(\varepsilon), n \in \mathbb{Z}_{-}, \quad \mathrm{h}_{m}^{0, \mathrm{u}}(\varepsilon)=q_{\mathrm{h}}^{\mathrm{u}, \varepsilon} \in W_{\varepsilon, \text { loc }}^{\mathrm{u}} \\
\mathrm{h}_{m}^{n}(0), n \in \mathbb{Z}, \quad \mathrm{h}_{m}^{0}(0)=q_{\mathrm{h}} \\
\mathrm{h}_{m}^{n, \mathrm{~s}}(\varepsilon), n \in \mathbb{Z}_{+}, \quad \mathrm{h}_{m}^{0, \mathrm{~s}}(\varepsilon)=q_{\mathrm{h}}^{\mathrm{s}, \varepsilon} \in W_{\varepsilon, \text { loc }}^{\mathrm{s}}
\end{gathered}
$$

We define the following distance between $q_{\mathrm{h}}^{\mathrm{u}, \varepsilon}, q_{\mathrm{h}}^{\mathrm{s}, \varepsilon}$ along the tangent vector $\nu_{0}$ at the point $\mathrm{h}_{m}^{0}$ :

$$
\begin{aligned}
\mathrm{d}=\operatorname{distance}\left(q_{\mathrm{h}}^{\mathrm{u}, \varepsilon}, q_{\mathrm{h}}^{\mathrm{s}, \varepsilon}\right) & =\sum_{m=0}^{M-1} \nu_{0}\left(\mathrm{~h}_{m}^{0}\right) \cdot\left(\mathrm{h}_{m+1}^{0, \mathrm{u}}(\varepsilon)-\mathrm{h}_{m+1}^{0, \mathrm{~s}}(\varepsilon)\right) \\
& =\left\langle\nu_{0}, \mathrm{~h}_{m}^{0, \mathrm{u}}(\varepsilon)-\mathrm{h}_{m}^{0, \mathrm{~s}}(\varepsilon)\right\rangle=\mathcal{D}^{\mathrm{u}}(\varepsilon)-\mathcal{D}^{\mathrm{s}}(\varepsilon)
\end{aligned}
$$

where the tangent vector $\nu_{0}$ is defined by the gradient of the function $\Delta$ (c.f (3.24)). We define

$$
\begin{aligned}
& \mathcal{D}^{\mathrm{s}}(\varepsilon)=\left\langle\nu_{0}\left(\mathrm{~h}_{m}^{0}\right), \mathrm{h}_{m}^{0, \mathrm{~s}}(\varepsilon)-\mathrm{h}_{m}^{0, \mathrm{u}}(\varepsilon)\right\rangle \\
& \mathcal{D}^{\mathrm{u}}(\varepsilon)=\left\langle\nu_{0}\left(\mathrm{~h}_{m}^{0}\right), \mathrm{h}_{m}^{0, \mathrm{u}}(\varepsilon)-\mathrm{h}_{m}^{0, \mathrm{u}}(\varepsilon)\right\rangle
\end{aligned}
$$

The orbits $\mathrm{h}_{m}^{n}, \mathrm{~h}_{m}^{n, \mathrm{u}}, \mathrm{h}_{m}^{n, \mathrm{~s}}$ fulfil the equations:

$$
\begin{aligned}
\mathrm{h}_{m}^{n+1} & =\mathbf{F}\left(\mathrm{h}_{m}^{n-1}, \mathrm{~h}_{m+1}^{n}, \mathrm{~h}_{m-1}^{n}\right) \\
\mathrm{h}_{m}^{n+1, \mathrm{u}}(\varepsilon) & =\mathbf{F}\left(\mathrm{h}_{m}^{n, \mathrm{u}}(\varepsilon)\right)+\varepsilon \mathbf{G}\left(\mathrm{h}_{m}^{n, \mathrm{u}}(\varepsilon)\right) \\
\mathrm{h}_{m}^{n+1, \mathrm{~s}}(\varepsilon) & =\mathbf{F}\left(\mathrm{h}_{m}^{n, \mathrm{~s}}(\varepsilon)\right)+\varepsilon \mathbf{G}\left(\mathrm{h}_{m}^{n, \mathrm{~s}}(\varepsilon)\right)
\end{aligned}
$$

where $\mathbf{F}, \mathbf{G}$ defined explicitly in (4.11). We study the motion on the unstable manifold. From (4.17) we have

$$
\mathrm{y}_{m}^{n+1}=\left.\frac{\mathrm{d}}{\mathrm{d} \varepsilon} h_{m}^{n+1, \mathrm{u}}(\varepsilon)\right|_{\varepsilon=0}=\mathrm{DF}\left(\mathrm{h}_{m}^{n, \mathrm{u}}\right) \mathrm{y}_{m}^{n, \mathrm{u}}+\mathbf{G}\left(\mathrm{h}_{m}^{n, \mathrm{u}}\right)
$$

then:

$$
\left\langle\nu_{m}^{n+1}, \mathrm{y}_{m}^{n+1}\right\rangle=\left\langle\nu_{m}^{n+1}, \mathrm{DF}\left(\mathrm{h}_{m}^{n}\right) \mathrm{y}_{m}^{n}\right\rangle+\left\langle\nu_{m}^{n+1}, \mathbf{G}\left(\mathrm{h}_{m}^{n, \mathrm{u}}\right)\right\rangle
$$

Using the integrability of the unperturbed system $\mathbf{F}$ and the mathematical induction, we obtain:

$$
\left\langle\nu_{m}^{0}, \mathrm{y}_{m}^{0, \mathrm{u}}\right\rangle=\sum_{n=-\infty}^{0} \sum_{m=0}^{M-1} \nu_{m}^{n} \mathbf{G}\left(\mathrm{h}_{m+1}^{n-1}\right)
$$


and similar for the stable manifold

$$
\left\langle\nu_{m}^{0}, \mathrm{y}_{m}^{0, \mathrm{~s}}\right\rangle=\sum_{n=1}^{\infty} \sum_{m=0}^{M-1} \nu_{m}^{n} \mathbf{G}\left(\mathrm{h}_{m+1}^{n-1}\right)
$$

Then

$$
\mathrm{d}=\varepsilon\left(\mathcal{D}^{\mathrm{u}}(0)-\mathcal{D}^{\mathrm{S}}(0)\right)+\mathcal{O}\left(\varepsilon^{2}\right)=\varepsilon \mathrm{M}_{\mathcal{F}}+\mathcal{O}\left(\varepsilon^{2}\right)
$$

where

$$
\mathrm{M}_{\mathcal{F}}:=\sum_{n \in \mathbb{Z}} \sum_{m=0}^{M-1} \nu_{m}^{n}\left(\mathrm{~h}_{m}^{n}\right) \cdot \mathbf{G}\left(\mathrm{h}_{m}^{n-1}\right)
$$

with $\nu_{m}^{n}$ defined in $(3.26 \mathrm{a}, 3.26 \mathrm{~b})$, evaluated on the homoclinic orbit $\mathrm{h}_{m}^{n}$.

The Mel'nikov function (4.21) becomes:

$$
\mathrm{M}_{\mathcal{F}}(\mathrm{x}, \tau ; h, M)=\mathrm{bS}_{1}+\mathrm{cS}_{2}
$$

with

$$
\begin{aligned}
& \mathrm{S}_{1}(\mathrm{x}, \tau ; h, M)=\sum_{n \in \mathbb{Z}} \sum_{m=0}^{M-1} \frac{\delta \mathcal{F}}{\delta \theta_{m}^{n}}\left(\mathrm{~h}_{m}^{n}\right) \cdot \mathrm{h}_{m}^{n-1} \\
& \mathrm{~S}_{2}(\mathrm{x}, \tau ; h, M)=\sum_{n \in \mathbb{Z}} \sum_{m=0}^{M-1} \frac{\delta \mathcal{F}}{\delta \theta_{m}^{n}}\left(\mathrm{~h}_{m}^{n}\right) \cdot \frac{\mathrm{h}_{m+1}^{n-1}-2 \mathrm{~h}_{m}^{n-1}+\mathrm{h}_{m-1}^{n-1}}{h^{2}}
\end{aligned}
$$

Setting $\mathrm{M}_{\mathcal{F}}=0$ we obtain an algebraic equation which describe a two-dimensional surface on the external parameter space $(b, c)$ :

$$
\mathrm{bS}_{1}+\mathrm{cS}_{2}=0
$$

We make the following observation, the only singularities of the homoclinic orbits $\Theta_{m}^{n}\left(=\mathrm{h}_{m}^{n}\right)$ are simple poles at any point $\tau_{\theta} \in \frac{\mathrm{i} \pi}{2}+\mathrm{i} \pi \mathbb{Z}$ and therefore $\Theta_{m}^{n}$ is analytic at $\tau_{\theta}+h k$ for $k \in \mathbb{Z} \backslash\{0\}$.

It is important to keep in mind that our model admits an invariant subspace $\Pi$ and the persistent homoclinic orbit will leave the saddle point $\hat{\theta}_{m}^{n}(\varepsilon)$ near $\Pi$, rapidly fly away and return along a global orbit which is close to one of the integrable homoclinic orbit and must approach the saddle point. In order to investigate this fact, we focus our attention to the dynamics of perturbed discrete pendulum in $\Pi$. The dynamics of dDSG on $\Pi$ is governed by the following standard map (so-called discrete pendulum):

$$
\theta^{n+1}=-\theta^{n-1}+2 \theta^{n}-4 \operatorname{Arg}\left(1+\frac{h^{2}}{4} \mathrm{e}^{\mathrm{i} \theta^{n}}\right)+\varepsilon \mathrm{b} \theta^{n}
$$

or

$$
\mathrm{f}_{\Pi}(x, y)=\left(y,-x+2 y-4 \operatorname{Arg}\left(1+\frac{h^{2}}{4} \mathrm{e}^{\mathrm{i} y}\right)\right)+\varepsilon(0, \mathrm{~b} y):=\mathrm{f}_{0}+\varepsilon \mathrm{f}_{1}
$$

We observe that the mapping $\mathrm{f}_{0}$ is an area preserving with the following generating function

$$
\mathcal{S}_{0}(x, X)=-x X+X^{2}+\tilde{F}(X+\sigma)+\tilde{F}(\sigma-X)
$$

with

$$
\tilde{F}(x)=2 \mathrm{i} \int_{-\infty}^{x} \ln \left(1+\mathrm{e}^{\mathrm{i} \xi}\right) \mathrm{d} \xi, \quad \mathrm{e}^{\mathrm{i} \sigma}=h^{2} / 4
$$


The solution $\hat{\theta}^{n}=\pi$ is a saddle fixed point of the unperturbed map $\mathrm{f}_{0}$, since the residues $\mathcal{R}$ of the Jacobian matrix $\mathrm{Df}_{0}(\pi)$ passes through zero:

$$
\mathcal{R}=\frac{1}{4}\left(2-\operatorname{traceDf}_{0}(\pi)\right)=\frac{h^{2}}{h^{2}-4}<0, \quad 0<h<1
$$

Another feature of the discrete pendulum is that it admits an one-parameter $(\tau \in \mathbb{R})$ family of homoclinic orbits to the saddle point $\hat{\theta}^{n}=\pi$ :

$$
\begin{aligned}
\theta^{n}(\tau) & =\pi+4 \tan ^{-1}(\operatorname{sinhw} \cos \mathrm{x} \operatorname{sech}(n \mathrm{w} h+\tau)) \\
\theta^{n+1}(\tau) & =\pi+4 \tan ^{-1}(\sinh \mathrm{w} \cos \mathrm{xech}((n+1) \mathrm{w} h+\tau))
\end{aligned}
$$

with

$$
\mathrm{w}=\sinh ^{-1} \mu \quad \text { and } \quad \mu:=h^{2}\left(\frac{h^{2}}{4}-1\right)^{-1}
$$

One can verify after some manipulations that the expression (4.26) satisfies the map $f_{0}$ and

$$
\lim _{n \rightarrow \pm \infty} \theta^{n}=\pi
$$

with $\pi$ is a saddle point of the map $f_{0}$. A third aspect of the discrete pendulum system directly related to the integrability. The unperturbed map (4.24) has the following integral of motion

$$
H\left(\theta^{n}, \theta^{n-1}\right)=2\left(\cos \theta^{n}+\cos \theta^{n-1}\right)+\frac{h^{2}}{4} \cos \left(\theta^{n}+\theta^{n-1}\right)+\frac{4}{h^{2}} \cos \left(\theta^{n}-\theta^{n-1}\right)
$$

Moreover, the dynamics of (4.24) with $\varepsilon$ small is $\varepsilon$-close to the dynamics of discrete pendulum. These properties of the map (4.24) are inportant to establish the persistence of homoclinic orbits for the dDSG equation.

We have proved that there are generic intersection between $W^{\mathrm{u}}\left(\hat{\theta}_{m}^{n}(\varepsilon)\right)$ and $W^{\mathrm{s}}\left(\mathcal{M}_{\varepsilon}\right) \subset \mathcal{M}_{\varepsilon}$, so that for external parameters (b,c) in a fixed open set there are orbits $\mathrm{h}_{m}^{n}(\varepsilon)$ which tend to $\hat{\theta}_{m}^{n}(\varepsilon)$ as $n \rightarrow-\infty$ and approach $\mathcal{M}_{\varepsilon}$ in forward time $n \rightarrow+\infty$. Now, we prove that these orbits in forward time also tend to $\hat{\theta}_{m}^{n}(\varepsilon)$.

Let

$$
\begin{aligned}
\left.W^{\mathrm{s}}\left(\hat{\theta}_{m}^{n}(\varepsilon)\right)\right|_{\Pi} & =\Pi \cap W^{\mathrm{s}}\left(\hat{\theta}_{m}^{n}(\varepsilon)\right) \\
\left.W^{\mathrm{s}, \mathrm{u}}\left(\hat{\theta}_{m}^{n}(\varepsilon)\right)\right|_{\mathcal{M}_{\varepsilon}} & =\mathcal{M}_{\varepsilon} \cap W^{\mathrm{s}, \mathrm{u}}\left(\hat{\theta}_{m}^{n}(\varepsilon)\right)
\end{aligned}
$$

and from the linearized analysis we have $\left.\operatorname{dim} W^{\mathrm{s}, \mathrm{u}}\left(\hat{\theta}_{m}^{n}(\varepsilon)\right)\right|_{\Pi}=1,\left.\operatorname{codim} W^{\mathrm{s}}\left(\hat{\theta}_{m}^{n}(\varepsilon)\right)\right|_{\mathcal{M}_{\varepsilon}}=1$ in $\mathcal{M}_{\varepsilon}$. Let $q^{\mathrm{S}, \varepsilon}$ denote the intersection point of the orbit $\mathrm{h}_{m}^{n}(\varepsilon)$ with the boundary of the set $U$ ( $U$ be a neighborhood of the phase space of (4.24), then $q^{\mathrm{S}, \varepsilon} \in \partial U \cap W^{\mathrm{S}}\left(\mathcal{M}_{\varepsilon}\right)$, with projection point $\pi\left(q^{\mathrm{s}, \varepsilon}\right) \in \mathcal{M}_{\varepsilon}$. From the invariant manifolds theory, the points $q^{\mathrm{S}, \varepsilon}$ and $\pi\left(q^{\mathrm{S}, \varepsilon}\right)$ will approach the same point in forward time. Also, we have

$$
\operatorname{distance}\left(q^{\mathrm{s}, \varepsilon}, \pi\left(q^{\mathrm{s}, \varepsilon}\right)\right)=\mathcal{O}(\varepsilon)
$$

We measure the distance between the projection point $\pi\left(q^{\mathrm{s}, \varepsilon}\right)$ and the point $\left.q_{\Pi}^{\mathrm{u}, \varepsilon} \in W^{\mathrm{u}}\left(\hat{\theta}_{m}^{n}(\varepsilon)\right)\right|_{\Pi}$

$$
d:=\operatorname{distance}\left(\pi\left(q^{\mathrm{s}, \varepsilon}\right), q_{\Pi}^{\mathrm{u}, \varepsilon}\right)=\varepsilon \mathrm{M}_{H}(\tau, w)+\mathcal{O}\left(\varepsilon^{2}\right)
$$

The Mel'nikov sum $\mathrm{M}_{H}$ is built with the constant of motion $H$ of discrete pendulum, (c.f (4.28)):

$$
\mathrm{M}_{H}(\tau ; h)=\sum_{n \in \mathbb{Z}} \mathrm{g}(\tau+n \mathrm{w} h)
$$


where

$$
\begin{gathered}
\mathrm{g}:=\mathrm{b} \frac{\partial H}{\partial \theta^{n}}(\theta(\tau+n \mathrm{w} h)) \theta(\tau+n h \mathrm{w}) \\
\frac{\partial H}{\partial \theta^{n}}=-2 \sin \theta^{n}-\frac{h^{2}}{4} \sin \left(\theta^{n}+\theta^{n-1}\right)-\frac{4}{h^{2}} \sin \left(\theta^{n}-\theta^{n-1}\right)
\end{gathered}
$$

and $\theta^{n}(\tau)$ is given in (4.26). The above sum reduces to compute the residues of the function g. In particular, the function that plays a significant role in the computation of the infinite sum (4.30) is a complex function $\chi$ satisfying the following properties: $\chi$ is meromorhic in $\mathbb{C}$, is $T$ i-periodic and w-periodic and the set of poles of $\chi$ is $w \mathbb{Z}+T \mathrm{i} \mathbb{Z}$, being all of them simple and of residue 1 . Also, the function $g$ appear in formula (4.30) verifying: (i) is analytic in $\mathbb{R}$ and has only isolated singularity in $\mathbb{C}$; is $T$ i-periodic for $T>0$, (iii) and fulfils $|\mathrm{g}(\tau)| \leq \operatorname{Aexp}[-c|\operatorname{Re} \tau|]$ when $|\operatorname{Re} \tau| \rightarrow \infty$ for some constants $\mathrm{A}, c>0$. Then, the Mel'nikov function $\mathrm{M}_{H}$ is analytic in $\mathbb{R}$, has only isolated singularities in $\mathbb{C}$ and is doubly periodic with periods $h, T \mathrm{i}$. Moreover $\mathrm{M}_{H}(\tau)$ can be expressed by the following sum:

$$
\mathrm{M}_{H}(\tau ; h)=-\sum_{z \in \mathcal{J}} \operatorname{res}\left(\chi_{T} \mathrm{~g} ; z\right)
$$

where

$$
\mathcal{J}:=\{z \in \mathbb{C}: 0<\operatorname{Im} z<T\}
$$

The two condition of Melnikov function $\mathbf{M}=\left(\mathrm{M}_{\mathcal{F}}, \mathrm{M}_{H}\right)$

$$
\mathrm{M}_{\mathcal{F}}=0, \quad \text { and } \quad \mathrm{M}_{H}=0
$$

will select isolated values of two variables $\mathrm{x}, \tau$ (x, $\tau$ parametrize the homoclinic orbits). This will certainly be the case if, in addition to satisfying (4.33), $\mathrm{x}$ and $\tau$ also satisfy the transversality condition:

$$
\operatorname{det} D_{(\mathrm{x}, \tau)} \mathbf{M} \neq 0
$$

where $D$ the Jacobian of the Mel'nikov function. If the condition (4.33) is augmented by this transversality condition then the homoclinic orbits corresponding to these selected isolated values do in fact persist for $0<\varepsilon \ll 1, \mathrm{w}_{1}, \mathrm{w}_{2}$ given by $(4.27)$ and $\mathrm{b}, \mathrm{c} \in \mathbb{R}$. Thus there exists exactly transversal intersection point and the perturbed orbits $\mathrm{h}_{m}^{n}(\varepsilon)$ tend to $\hat{\theta}_{m}^{n}(\varepsilon)$ as $n \rightarrow+\infty$. We can now state the result.

Proposition 2 Consider the near-integrable dDSG equation (2.8) with periodic boundary conditions (2.6). Let $M$ be an even fixed positive integer and $h:=2 \pi / M<1$ be a constant real number. Then, there exists $\varepsilon_{0}>0$ such that, for any fixed parameters $\left\{\mathrm{w}_{1}, \mathrm{w}_{2}, \mathrm{~b}, \mathrm{c}, \varepsilon\right\}$ there are isolated values $\mathrm{x}_{0}, \tau_{0}$ of two variables $\mathrm{x}, \tau$ at the Melnikov functions $\mathrm{M}_{\mathcal{F}}, \mathrm{M}_{H}$ satisfy the following condtionions

$$
\begin{gathered}
\mathrm{M}_{\mathcal{F}}=0, \quad \text { and } \quad \mathrm{M}_{H}=0 \\
\operatorname{det} D_{(\mathrm{x}, \tau)} \mathbf{M} \neq 0
\end{gathered}
$$

The system (2.8) admits a homolcinic orbits $\theta_{m}^{n}(\varepsilon)$ that is doubly asymptotic to the fixed point $\hat{\theta}_{m}^{n}(\varepsilon) \in \Pi$.

\section{Conclusions}

We have developed a geometric method of studying the behaviour of homoclinic solutions of double discrete sine-Gordon equation with periodic boundary conditions under small dissipative perturbations. We choose a class of dDSG equation to illustrate between the methods of $\mathrm{P} \Delta$ Es and those of dynamical 
systems theory (symplectic mappings) by which these properties can be understood mathematically. Specifically, for the study of global behaviour for soliton $\mathrm{P} \Delta \mathrm{Es}$, we advocate implementary int uition from the theory of dynamical systems with methods natural from the P $\Delta$ Es. We found an analytic expression of homoclinic orbits for dDSG under periodic boundary conditions through Bäcklund transformation and Lax pair formalism. We have shown that these orbits persist under dissipative perturbations through the Mel'nikov criteria for discrete dynamical systems. Moreover, our results should be interested in the construction of new discrete surface and how these new geometric objects can be obtained through numerical simulations and a comparison with the well-known discrete surfaces will be important. In the future works, we will study the geometric principles and the homoclinic structure in other important class of lattice equations obtained by extending the dDSG equation to higher order partial difference systems.

Acknowledgements The author is indebted to F W Nijhoff for stimulating discussions and R S MacKay, V G Papageorgiou for useful comments. 


\section{References}

[1] Ablowitz M J and Ladik J F, 1976 J. Math. Phys 17, 1011.

[2] Ablowitz M J, Herbst B and Shober C, 1996 J Comp. Phys. 126 299-314 and 1997 J Comp. Phys. $131354-367$.

[3] Bobenko A, Kutz N and Pinkal U, 1993 Phys. Lett. A 177, 399-404.

[4] Bobenko A and Pinkal U, 1996 J. Diff. Geometry 43, 527-611.

[5] Capel H W, Nijhoff F W and Papageorgiou V G, 1991 Phys. Lett. A 155377.

[6] Ercolani N, Forest M G and McLaughlin D W, 1990 Phys. D 43 349-384.

[7] Faddeev L D and Volkov A Yu, 1994 Lett. Math. Phys. 32 125-135.

[8] Glasser M I, Papageorgiou V and Bountis T, 1989 SIAM J. Appl. Math. 49(3), 692-703.

[9] Haller G, 1999 Chaos near resonance, Applied Mathematical Sciences, 138. Springer-Verlag, New York.

[10] Hirota R, 1977 J. Phys. Soc. Japan 43, 2079.

[11] Kutz N, 1999 Comm. Math. Phys 204, 115-136.

[12] Li Y and McLaughlin D W, 1994 Comm. Math. Phys. 162, 1, 175-214.

[13] Li Y, McLaughlin D W, Shatah J and Wiggins St, 1996 Comm Pure and Appl Math, XLIX $1175-1255$.

[14] MacKay R S and Aubry S, 1994 Nonlinearity 7, 1263-1243.

[15] McLaughlin D W and Overman E A.II, 1995 Surveys in Applied Mathematics, 1, 83-203, Plenum, New York.

[16] McLaughlin D W and Shatah J, 1998 Recent advances in partial differential equations, Venice 1996, 281-299, Proc. Sympos. Appl. Math., 54, AMS Providence, RI.

[17] Mel'nikov V K, 1963 Trans. Moscow Math., 12 1-57.

[18] Nijhoff F W and Capel H W, 1995 Acta Applicandae Mathematicae 39 133-158.

[19] Orfanidis S J, 1978 Phys. Rev D 18 (10)3822-27, 3828-32, and 1980 Phys. Rev D 21 (6) 1507-12.

[20] Quispel G R W, Capel H W, Papageorgiou V G and Nijhoff F W, 1991 Physica A173 243.

[21] Rothos V M, 1999 J. Phys. A: Math. and Gen., 32, (36), 6409-6422 and 2000 in Proc. of Workshop Nonlinearity, Integrability and all that 20 years after NEEDS'r9, Eds. M. Boiti et al, 402-408 World Scientific.

[22] Matveev V and Salle M A, 1991 Darboux Transformations and Solitons Vol 5 Springer Series in Nonlinear Dynamics-Springer Verlag NY

[23] Toda M, 1975 J. Phys. Soc. Jap 22, 431. 